\title{
Loss analysis of air-core photonic crystal fibers
}

\author{
Yong Xu and Amnon Yariv \\ Department of Applied Physics, MS 128-95, California Institute of Technology, Pasadena, California 91125
}

Received April 7, 2003

\begin{abstract}
By using a multipole moment approach, we analyze the loss of an air-core photonic crystal fiber and demonstrate that it is possible reduce the transmission loss that is due to photon radiation leakage through the photonic crystal cladding to a level below $0.01 \mathrm{~dB} / \mathrm{km}$, with eight rings of air holes. An analogy is drawn between air-core photonic crystal fiber modes and Bragg fiber modes. The influence of material absorption in the silica glass is discussed. (c) 2003 Optical Society of America
\end{abstract}

OCIS codes: $060.2280,230.1480$.

Recently the use of Bragg reflection to confine light in an air-core fiber has attracted considerable attention. $^{1-8}$ Some advantages of air-core fibers are their potential to achieve propagation losses of less than $0.2 \mathrm{~dB} / \mathrm{km}$ (the current value of telecommunication fibers) and to greatly increase the power threshold for the onset of nonlinear effects. The air-core fibers considered in the literature can be generally classified into two types: photonic crystal fibers ${ }^{2-5}$ (PCFs) and Bragg fibers. ${ }^{1,6,7,8}$ Some important advantages of PCFs are the excellent material properties of the silica glass and the compatibility of PCFs with the current optical fiber manufacturing technologies. Yet Bragg reflection in an air-core PCF with a finite number of cladding air holes always leads to some radiation leakage of light from the fiber core, which is difficult to calculate owing to the structural complexity of the PCF. The method of plane-wave expansion in a supercell, ${ }^{3}$ because of an artificially imposed periodic boundary condition, cannot give the propagation loss. Many other numerical approaches do not have the numerical precision to resolve propagation loss of the order of decibels per kilometer or less. In this Letter we use newly developed multipole moment approach $^{4,9}$ to analyze the modal loss of an aircore PCF.

As shown in Fig. 1(a), the air-core PCF is formed by replacing the inner seven air cylinders in a triangular lattice with one bigger air core of radius $r_{\mathrm{co}}=$ $3.106 \mu \mathrm{m}$. The cladding air cylinders have a lattice pitch $\Lambda=2.875 \mu \mathrm{m}$, a radius $r=1.394 \mu \mathrm{m}$, and a finite number of rings $N_{\text {ring. The index of the silica }}$ glass takes the value 1.45. According to group theory, the symmetry operations of the PCF forms a $C_{6 v}$ point group, and, as a result, the guided modes can be divided into eight classes. ${ }^{10}$ For each symmetry class, the azimuthal dependence of the guided modes is given by $\sum_{m} f_{m}(r) \exp (i m \theta)$, with $m=6 n \pm m_{0}$, where $n=0, \pm 1, \pm 2, \ldots$ and $m_{0}$ is an integer from 0 and 3 that depends on the symmetry class. ${ }^{10}$ In this Letter we consider only the guided modes that are confined within the air core and are dominated by the components with small $m$. As a result, we limit our discussion to the guided modes that belong to symmetry classes 1 and 2 (which are nondegenerate with $m_{0}=0$ and to those of symmetry classes 3 and 4 (which are degenerate with $\left.m_{0}=1\right) .{ }^{10}$
In Fig. 1(b) we give the $\Gamma M$ finger diagram for the fiber shown in Fig. 1(a), and the guided modes with symmetry classes $1-4$. We calculated the $\Gamma M$ finger diagram by counting the number of photonic crystal cladding modes with the Bloch vector along the $\Gamma M$ direction in reciprocal space. ${ }^{4}$ In Fig. 2 we plot the $z$ component of the Poynting vector $\left(P_{z}\right)$ of the class 1 mode, which is clearly dominated by the $m=0$ component. We can denote the class 1 mode as the TM-like
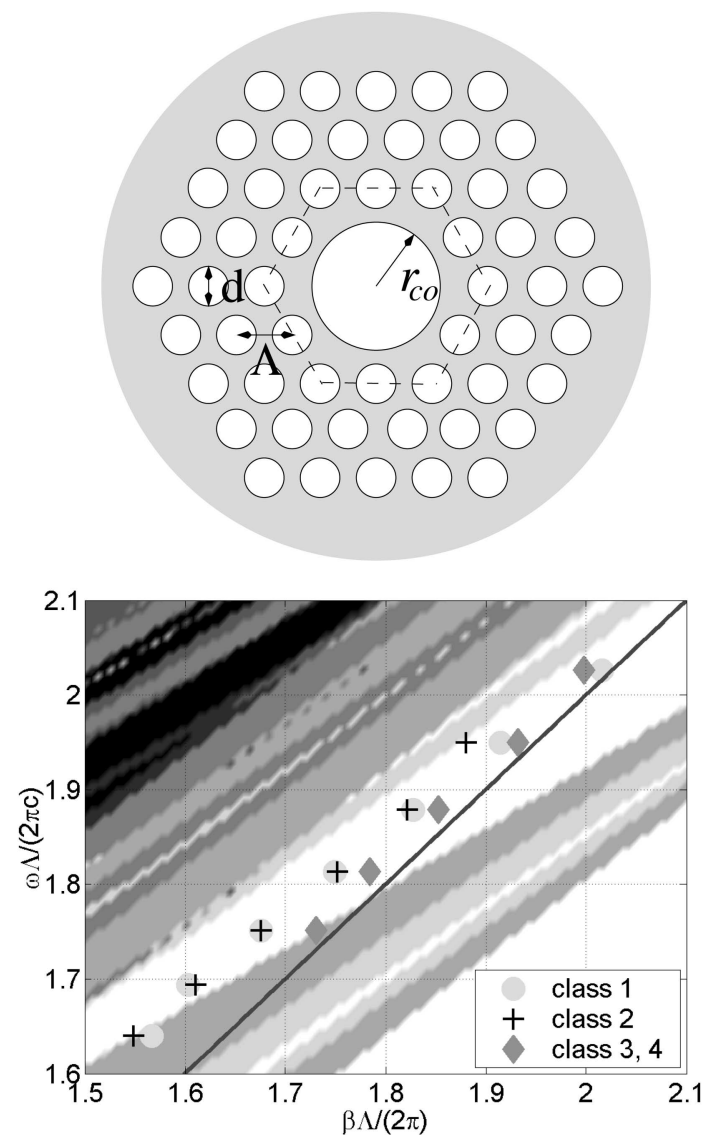

Fig. 1. (a) Cross section of an air-core photonic crystal fiber. The dashed line denotes a ring of air cylinders. (b) $\Gamma M$ finger diagram of the triangular lattice cladding (with the color density proportional to the number of propagating states) and dispersions of the guided modes with different symmetry. The straight line is the light line, which is given by $\beta=\omega / c$. 

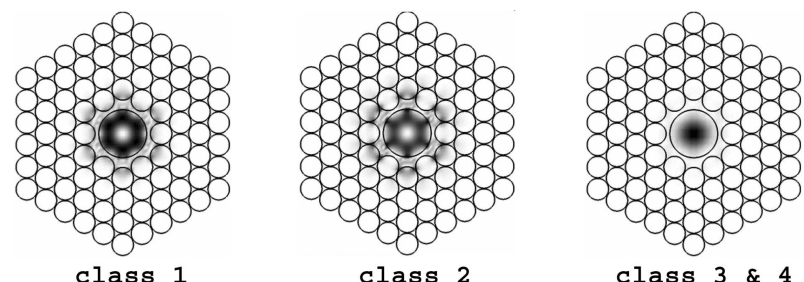

Fig. 2. Distribution of the $z$ component of the Poynting vector.

mode, because the $m=0$ component consists entirely of the $E_{z}, H_{\theta}$, and $E_{r}$ field components and resembles the TM mode of a Bragg fiber., ${ }^{1,6}$ The class 2 mode, as shown in Fig. 2, is also dominated by the $m=0$ component, with a small part being the $m=6$ component. The $m=0$ component of the class 2 mode resembles the TE mode of a Bragg fiber $^{1,6}$ and can be similarly denoted a TE-like mode. The power flux of symmetry class 3 and class 4 modes, however, clearly resembles the fundamental $\mathrm{HE}_{11}$ mode of a step-index fiber (therefore we denote it HE-like) and consists almost entirely of the $m=1$ component, as required by group theory.

For an air-core PCF, as in the case of Bragg fibers, ${ }^{8}$ propagation loss $\alpha_{s}$ should decreases exponentially as the number of air cylinder rings $N_{\text {ring }}$ increases: $\alpha_{s} \propto$ $1 / D_{s} N_{\text {ring }}$, where $s$ refers to the symmetry class of the mode and $D_{s}$ is a constant. In Figs. 3(a)-3(c) we show the modal propagation loss as a function of $N_{\text {ring }}$ at three wavelengths, together with the exponential fit and the exponential constant $D_{s}$. In every case, the exponential fit gives an excellent approximation of the modal loss. As a result, we can extrapolate the modal loss to a PCF with eight rings of air holes. We find that at $1.53 \mu \mathrm{m}$ the loss of the class 3 mode drops below the level of $0.01 \mathrm{~dB} / \mathrm{km}$, a value 20 times below that of current telecommunication fibers. This value is calculated for an ideal PCF, i.e., without any air-hole deformation or fiber nonuniformity, and can be regarded as a lower limit for the propagation loss in a particular PCF. This lower limit is much smaller than the current experimental record of $13 \mathrm{~dB} / \mathrm{km},{ }^{5}$ which suggests that the main limiting factor in lowering propagation loss is imperfection in the PCF.

In previous calculations we ignored the modal attenuation that is due to optical absorption in the silica glass. However, as the loss that is due to photon leakage approaches the level of loss in a conventional silica fiber, it becomes necessary to include the absorption in the silica glass, which can be taken into account from the following simple considerations. We introduce a small imaginary part $\operatorname{Im}\left(n_{\text {glass }}\right)$ into the refractive index of the silica glass and calculate the effective index of the guided mode, $n_{\text {eff }}$, defined as the complex propagation constant divided by the vacuum wave vector. The imaginary part of the effective index, $\operatorname{Im}\left(n_{\text {eff }}\right)$, should have a linear dependence on $\operatorname{Im}\left(n_{\text {glass }}\right)$, whose linear coefficient tells us the percentage of the optical field in the silica glass. We apply this procedure to the fiber illustrated in Figs. 3(a)-3(c). We choose three rings of air holes and use a wavelength of $1.53 \mu \mathrm{m}$. The results are shown in Fig. 3(d) and demonstrate excellent linear dependence between $\operatorname{Im}\left(n_{\text {eff }}\right)$ and $\operatorname{Im}\left(n_{\text {glass }}\right)$. From a linear fit of the results we find that $\sim 13 \%$ of the optical field falls inside the silica glass. Therefore, for the class 3 mode we can expect that the absorption loss that is due to the silica glass will be of the order of $0.03 \mathrm{~dB} / \mathrm{km}$, a value comparable with that of the photon leakage loss.

In the case of Bragg fibers, the propagation loss of the TE modes is well described by a formula derived from the intuitive picture of a plane wave bouncing between two planar Bragg reflectors. ${ }^{8}$ A similar result can be derived for the air-core PCF. First, we notice that the guided modes shown in Fig. 2 are all dominated by components with angular quantum number $m$ of either 0 or 1 . We can define an effective azimuthal wave vector $k_{\theta}=m / r_{\text {co }}$ at the interface between the air core and the photonic crystal cladding to characterize the angular variation of the electromagnetic field. For the $m=0$ and the $m=1 \mathrm{com}$ ponents at $\lambda=1.55 \mu \mathrm{m}$, the values of azimuthal wave vector $k_{\theta}$ normalized by vacuum wave vector $\omega / c$ are, respectively, 0 and 0.08 . Since $k_{\theta}$ is smaller than $\omega / c$, we can transform the problem of propagation of light
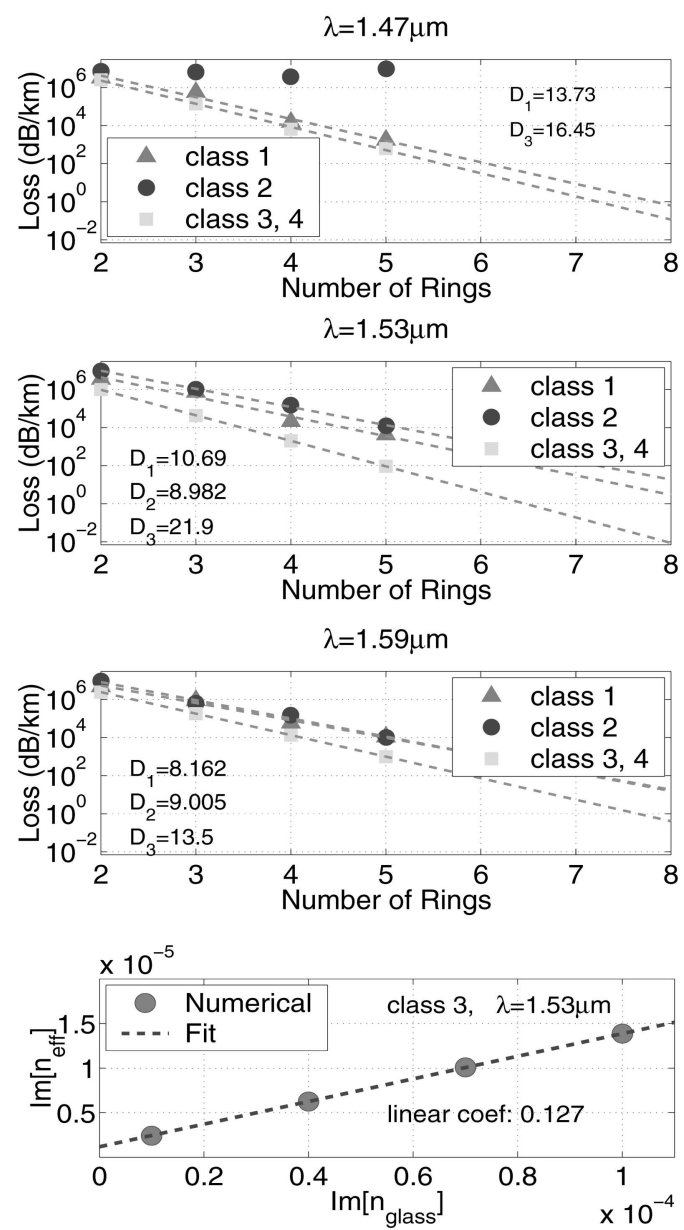

Fig. 3. (a)-(c) Propagation loss of the modes with symmetry classes 1-4. Dashed lines are fits assuming an exponential dependence. (d) Imaginary part of the modal effective index of the class 3 mode as a function of the imaginary part of the silica refractive index. 


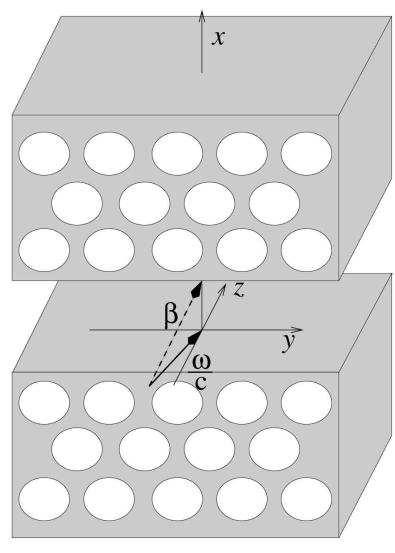

(a)

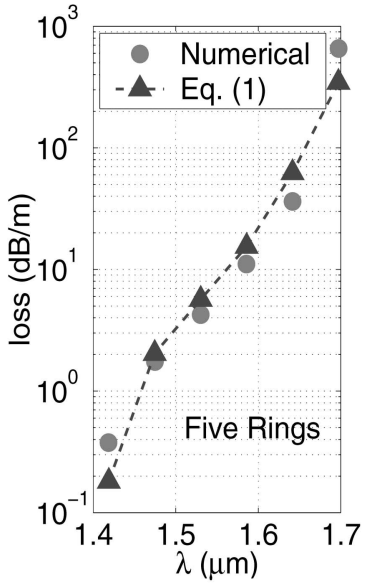

(b)
Fig. 4. (a) Wave zigzagging between two photonic crystal stacks. (b) Modal loss of the class 1 mode (TM-like) with five rings of air holes: circles, numerical results from the multipole expansion calculations; triangles were obtained from Eq. (1).

within the PCF air core into that of a wave bouncing between two parallel triangular lattice photonic crystal stacks with an incident angle $\cos ^{-1}(c \beta / \omega)$ [see Fig. $4(\mathrm{a})]$, where $\beta$ is the propagation constant. In this equivalent picture the incident photon wave vector is confined within the $x z$ plane. As a result, the corresponding photon Bloch vector within the triangular lattice cladding must be along the $\Gamma M$ direction in reciprocal space, which justifies the use of the $\Gamma M$ finger diagram in Fig. 1(b). We emphasize that analysis is different from most analyses of air-core PCFs in the literature, in which the use of the complete finger diagram that counts the PCF cladding modes along both the $\Gamma M$ and the $\Gamma K$ directions may lead to an underestimate of the frequency range of bandgap guiding. We point out that the existence of confined PCF modes outside the complete bandgap was shown in Fig. 2 of Ref. 4.

If we equate the propagation loss of a PCF with that of its equivalent photonic crystal stack shown in Fig. 4(a) and assume a transmission coefficient $\mathcal{T}$ for each bounce, we can approximate the PCF propagation loss (in decibels per kilometer) as

$$
\alpha=\frac{2.14 \times 10^{9}}{r_{\mathrm{co}}} \mathcal{T}\left[1-\left(\frac{\beta c}{\omega}\right)^{2}\right]^{1 / 2}[\mathrm{~dB} / \mathrm{km}]
$$

where $r_{\text {co }}$ is given in micrometers. Parameter $\mathcal{T}$ describes the transmission through the finite stack of the photonic crystal cladding and is calculated with a method described in Ref. 11, which is different from the previously used multipole moment method. Inasmuch as in general $\mathcal{T}$ depends on the polarization of the incident wave, we define the incident wave in Fig. 4(a) as TM (TE) polarized if the magnetic (the electric) field is along the $\hat{e}_{y}$ direction. In Fig. 4(b) we show the loss of the TM-like mode in an air-core PCF calculated from the multipole moment method as well as from the estimate given by Eq. (1) (assuming a TM incident wave). The fiber has five rings of air holes; the rest of the parameters are the same as those used in the previous calculations. The two results in Fig. 4(b) agree fairly well with each other, which not only validates the picture of a wave zigzagging within the air core but also confirms the use of the $\Gamma M$ finger diagram in the analysis of PCF, because the photon wave vector shown in Fig. 4(a) samples the Bloch wave vector only along the $\Gamma M$ direction. For the TE-like or the HE-like modes Eq. (1) does not work so well, a fact that can be attributed to the presence of both the TE component and the TM component. For the HE-like mode we use Eq. (1) to estimate the propagation loss for both the TE-polarized wave and the TM-polarized wave. Comparing these results with those obtained from the multipole moment method, we find that the loss of the HE-like mode largely follows the estimate that assumes a TM incident wave. In contrast, the loss of the TE-like modes varies from the estimates for which a TE-polarized wave is assumed and those for which a TM-polarized wave is assumed.

\section{References}

1. P. Yeh, A. Yariv, and E. Marom, J. Opt. Soc. Am. 68, 1196 (1978).

2. R. F. Cregan, B. J. Mangan, J. C. Knight, T. A. Birks, P. St. J. Russell, P. J. Roberts, and D. C. Allan, Science 285, 1537 (1999).

3. J. Broeng, S. E. Barkou, T. Søndergaard, and A. Bjarklev, Opt. Lett. 25, 96 (2000).

4. T. P. White, R. C. McPhedran, L. C. Botten, G. H. Smith, and C. M. de Sterke, Opt. Express 9, 721 (2001), http://www.opticsexpress.com.

5. N. Venkataraman, M. T. Gallagher, D. Müller, C. M. Smith, J. A. West, K. W. Koch, and J. C. Fajardo, presented at the European Conference on Optical Communications, Copenhagen, Denmark, September 12-18, 2002.

6. Y. Xu, R. K. Lee, and A. Yariv, Opt. Lett. 25, 1756 (2000).

7. S. D. Hart, G. R. Maskaly, B. Temelkuran, P. H. Prideaux, J. D. Joannopoulos, and Y. Fink, Science 296, 510 (2002).

8. Y. Xu, A. Yariv, J. G. Fleming, and S.-Y. Lin, Opt. Express 11, 1039 (2003), http://www.opticsexpress.com.

9. T. P. White, B. T. Kuhlmey, R. C. McPhedran, D. Maystre, G. Renversez, C. M. de Sterke, and L. C. Botten, J. Opt. Soc. Am. B 19, 2322 (2002).

10. P. R. McIssac, IEEE Trans. Microwave Theory Tech. MTT-23, 421 (1975)

11. G. H. Smith, L. C. Botten, R. C. McPhedran, and N. A. Nicorovici, Phys. Rev. E 66, 056604 (2002). 